and the conditions $(\beta)$ and $(\gamma)$ become, respectively,

$$
\left|\begin{array}{cc}
F_{\eta} & F_{x_{1}} \\
G_{\eta} & G_{x_{1}}
\end{array}\right|=0,
$$

$$
\left|\begin{array}{lll}
0 & F_{\eta} & F_{x_{1}} \\
F_{\eta} & F_{\eta z} & F_{z x_{1}} \\
G_{\eta} & G_{\eta z} & G_{z x_{1}}
\end{array}\right| \neq 0
$$

for the system of values

$$
x_{1}=a_{1}, \quad x_{2}=a_{2}, \cdots, x_{n}=a_{n} ; \eta=0, \quad z=c .
$$

Hence the hypothesis of Theorem I is satisfied for the equations $F=0, G=0$, and we have a unique solution of the form

$$
\eta=\eta\left(x_{1}, x_{2}, \cdots, x_{n}\right), \quad z=\psi\left(x_{1}, x_{2}, \ldots, x_{n}\right),
$$

such that

$$
0=\eta\left(a_{1}, a_{2}, \ldots, a_{n}\right), \quad c=\psi\left(a_{1}, a_{2}, \ldots, a_{n}\right) .
$$

Substituting the values of $\eta$ and $z$ in (19), we have the required solution for $y$.

If there is only one independent variable $x$, the conditions of Theorem II, for the system of values $x=y=z=0$, imply that the equations have the form

$$
\begin{aligned}
& f(x, y, z) \equiv[y-\lambda(z)] f_{1}(x, y, z)+x f_{2}(x, y, z)=0, \\
& g(x, y, z) \equiv[y-\lambda(z)] g_{1}(x, y, z)+x g_{2}(x, y, z)=0 .
\end{aligned}
$$

The curve defined by these equations consists of two branches passing through the origin. One branch is the curve $y=\lambda(z)$ in the $y z$-plane and is not expressible by equations of the form $y=\phi(x), z=\psi(x)$.

SHEFFIELd SCIENTIFIC School, May, 1910.

$$
\begin{aligned}
& \text { STURM'S METHOD OF INTEGRATING } \\
& d x / \sqrt{X}+d y / \sqrt{Y}=0 .
\end{aligned}
$$

BY PROFESSOR F. H. SAFFORD.

(Read before the American Mathematical Society, April 30, 1910.)

ONE of the simplest methods of obtaining the addition theorem for elliptic integrals of the first kind is based upon a method of integration which is usually referred to as Sturm's 
method, and was thus communicated by Liouville in the Comptes rendus, 1856, No. 21, page 988. But on page 1087 of the same volume Liouville stated that the method was due to Despeyrous, and in the Cours d'analyse of Ch. Sturm, Paris, 1877, volume 2, page 340 , the method is given in a note "par M. Despeyrous." The object of the present paper is to discuss certain details of Sturm's method and the extent to which it may be employed, without considering the addition theorems. The method is applicable to special types of the equation

$$
d x / \sqrt{ } f(x)+d y / \sqrt{ } f(y)=0,
$$

and since the variables in (1) are separated it can be integrated first term by term. By Sturm's method a second integral of (1) is sometimes obtainable by clearing of fractions and in most cases using also an integrating factor. Integration by parts is performed on each term of the new form of (1) and the two indicated integrations at this stage are to be combined under one integral sign; then if possible by the use of (1) this integrand is to be made to vanish. When this method is successful a comparison of the resulting integral with that first obtained leads to an addition theorem. Illustrations may be found in Byerly, Integral Calculus, page 235, and Durège, Elliptische Funktionen, 4th edition, page 101.

So far as the writer has discovered, in all cases where this method has been used the form of $f(x)$ has been considered known, and from the types $x^{2}, 1-x^{2},\left(1+x^{2}\right)^{2},\left(1-x^{2}\right)\left(1-k^{2} x^{2}\right)$ follow addition theorems for $\log x, \sin ^{-1} x, \tan ^{-1} x$, and $\operatorname{sn}^{-1} x$ respectively. The last two types require an additional integrating factor but the first two do not.

The following is from Schlömilch, Compendium der höheren Analysis, volume 2, page 326: "Es sei $* * *$

$$
d x / \sqrt{ }\left(1-x^{2}\right)+d y / \sqrt{ }\left(1-y^{2}\right)=0
$$

oder

mithin

$$
\sqrt{ }\left(1-y^{2}\right) d x+\sqrt{ }\left(1-x^{2}\right) d y=0,
$$

$$
\int \sqrt{ }\left(1-y^{2}\right) d x+\int \sqrt{ }\left(1-x^{2}\right) d y=\text { const. }
$$

Bei theilweiser Integration ist ferner 


$$
\begin{aligned}
& \int \sqrt{ }\left(1-y^{2}\right) d x=\sqrt{ }\left(1-y^{2}\right) x+\int \frac{x y d y}{\sqrt{ }\left(1-y^{2}\right)}, \\
& \int \sqrt{ }\left(1-x^{2}\right) d y=\sqrt{ }\left(1-x^{2}\right) y+\int \frac{y x d x}{\sqrt{ }\left(1-x^{2}\right)}
\end{aligned}
$$

mithin durch Addition

(6) $x \sqrt{ }\left(1-y^{2}\right)+y\left(1-x^{2}\right)+\int x y\left[\frac{d x}{\sqrt{ }\left(1-x^{2}\right)}+\frac{d y}{\sqrt{ }\left(1-y^{2}\right)}\right]=$ const.

Wegen Nro. (2) verschwindet das Integral und es bleibt einfacher

$$
x_{V}\left(1-y^{2}\right)+y \sqrt{ }\left(1-x^{2}\right)=c, * * * . "
$$

Though (7) is an integral of (2) it should be noticed that (3) is not an exact differential, and the integral in (6) for the analogous case $f(x)=x^{2}$ is a constant and does not vanish.

Schröter in the Zeitschrift für Mathematik und Physik, volume 17 (1872), page 508, avoids the difficulty by an inverse method which, applied to the example above, gives

$$
\begin{aligned}
d & {\left[x \sqrt{ }\left(1-y^{2}\right)+y V\left(1-x^{2}\right)\right] } \\
& =\left[\sqrt{ }\left(1-y^{2}\right) d x+\sqrt{ }\left(1-x^{2}\right) d y\right]-x y\left[\frac{d x}{\sqrt{ }\left(1-x^{2}\right)}+\frac{d y}{\sqrt{ }\left(1-y^{2}\right)}\right] \\
& =\left[\frac{d x}{\sqrt{ }\left(1-x^{2}\right)}+\frac{d y}{\sqrt{ }\left(1-y^{2}\right)}\right]\left[V\left(1-x^{2}\right) V\left(1-y^{2}\right)-x y\right] .
\end{aligned}
$$

Thus from (2) equation (8) becomes

$$
d\left[x \vee\left(1-y^{2}\right)+y V\left(1-x^{2}\right)\right]=0,
$$

whence the integral (7) follows. In this case no additional integrating factor is necessary.

The use of Sturm's method in integrating

$$
\frac{d x}{\sqrt{\left(1-x^{2}\right)\left(1-k^{2} x^{2}\right)}}+\frac{d y}{\sqrt{\left(1-y^{2}\right)\left(1-k^{2} y^{2}\right)}}=0
$$

has been called "elegant" by several writers who, however, have felt that the form of the integrating factor, after clearing of fractions, i. e., $1 /\left(1-k^{2} x^{2} y^{2}\right)$, is not obvious from the form of equation (10).

Schröter makes changes of variables in (10) and then shows how to determine the factor. But in the present paper the factor will be expressed, under certain sufficient conditions, as a 
function of $f(x)$ and $f(y)$ in the general type, equation (1), and then the corresponding form of $f(x)$ will be determined.

From (1), after supplying the unknown factor $F(x, y)$,

$$
\frac{V f(y) d x}{F}+\frac{\sqrt{ } f(x) d y}{F}=0 .
$$

The use of Sturm's method in its modified form gives

$$
\begin{aligned}
& d\left[\frac{x \sqrt{ } f(y)}{F}+\frac{y \sqrt{ } f(x)}{F}\right] \\
& =[x \sqrt{ } f(y)+y \sqrt{ } f(x)]\left[\frac{\frac{\partial F}{\partial x} d x+\frac{\partial F}{\partial y} d y}{-F^{2}}\right] \\
& +d x\left[\frac{\sqrt{ } f(y)+\frac{y f^{\prime}(x)}{2 \sqrt{ } f(x)}}{F}\right]+d y\left[\frac{\sqrt{ } f(x)+\frac{x f^{\prime}(y)}{2 \sqrt{ } f(y)}}{F}\right] \\
& =\frac{d x}{\sqrt{ } f(x)}\left[-\frac{x \sqrt{ } f(x) V f(y)}{F^{2}} \frac{\partial F}{\partial x}-\frac{y f(x)}{F^{2}} \frac{\partial F}{\partial x}\right. \\
& \left.+\frac{\sqrt{ } f(x) \sqrt{ } f^{\prime}(y)}{F}+\frac{y f^{\prime}(x)}{2 F}\right] \\
& +\frac{d y}{\sqrt{ } f(y)}\left[-\frac{y \sqrt{ } f(x) V f(y)}{F^{2}} \frac{\partial F}{\partial y}-\frac{x f(y)}{F^{2}} \frac{\partial F}{\partial y}\right. \\
& \left.+\frac{V f(x) V f^{\prime}(y)}{F}+\frac{x f^{\prime}(y)}{2 F^{\prime}}\right] \text {. }
\end{aligned}
$$

The last member of (12) will vanish in virtue of (1), provided the coefficients of $d x / \sqrt{ }(x)$ and $d y / \sqrt{ } f(y)$ are equal, and the sufficient conditions are these :

$$
\begin{gathered}
x \frac{\partial F}{\partial x}=y \frac{\partial F}{\partial y} \\
y f(x) \frac{\partial F}{\partial x}-\frac{y F f^{\prime}(x)}{2}=x f(y) \frac{\partial F}{\partial y}-\frac{x F f^{\prime}(y)}{2}
\end{gathered}
$$

From (13) it follows that $F$ is a function of the product $x y$, which is an important result and permits the reduction of (14) to

$$
2 x^{2} F^{\prime} f(y)-x F f^{\prime}(y)=2 y^{2} F^{\prime} f(x)-y F f^{\prime}(x)
$$


whence

$$
\frac{2 F^{\prime}}{F}=\frac{x f^{\prime}(y)-y f^{\prime}(x)}{x^{2} f^{\prime}(y)-y^{2} f(x)} .
$$

Since $F$ is a function of $x y$, the second member of (16) must also be a function of $x y$, and for proper forms of $f(x), F$ may be found from (16) by integration. An illustration is $f(x)=\left(1-x^{2}\right)\left(1-k^{2} x^{2}\right)$, then $F$ is $c\left(1-k^{2} x^{2} y^{2}\right)$.

The determination of the form of $f(x)$ is the next important step. Let the second member of (16) be called $V$; then employing the condition that $V$ shall be a function of $x y$, i. e.,

$$
x \frac{\partial V}{\partial x}=y \frac{\partial V}{\partial y},
$$

the result after some reduction is

$$
\begin{aligned}
& \frac{f(x) f^{\prime}(x)}{x^{3}}-\frac{f(y) f^{\prime}(y)}{y^{3}}+\frac{f(x) f^{\prime \prime}(x)}{x^{2}}-\frac{f(y) f^{\prime \prime}(y)}{y^{2}} \\
& +\frac{f^{\prime 2}(y)}{y^{2}}-\frac{f^{\prime 2}(x)}{x^{2}}+\frac{3 f^{\prime}(x) f(y)}{x y^{2}}-\frac{3 f^{\prime}(y) f(x)}{y x^{2}} \\
& +\frac{f(x) f^{\prime \prime}(y)}{x^{2}}-\frac{f(y) f^{\prime \prime}(x)}{y^{2}}=0 .
\end{aligned}
$$

When (17) is differentiated partially with respect to $x$ and $y$ in succession, the first six terms vanish and the remaining four give after reduction

$$
\begin{aligned}
& {\left[\frac{f^{\prime}(x)}{x^{2}}-\frac{2 f(x)}{x^{3}}\right]\left[f^{\prime \prime \prime}(y)-\frac{3 f^{\prime \prime}(y)}{x}+\frac{3 f^{\prime}(y)}{y^{2}}\right] } \\
= & {\left[\frac{f^{\prime}(y)}{y^{2}}-\frac{2 f(y)}{y^{3}}\right]\left[f^{\prime \prime \prime}(x)-\frac{3 f^{\prime \prime}(x)}{x}+\frac{3 f^{\prime}(x)}{x^{2}}\right] . }
\end{aligned}
$$

The variables in (18) may now be separated by division, and since the result is that a function of $x$ equals a function of $y$, each function is a constant, i. e.,

$$
\frac{f^{\prime \prime \prime}(x)-\frac{3 f^{\prime \prime}(x)}{x}+\frac{3 f^{\prime}(x)}{x^{2}}}{\frac{f^{\prime}(x)}{x^{2}}-\frac{2 f(x)}{x^{3}}}=4\left(a^{2}-1\right) .
$$


The form of the constant is chosen so that later results will be more simple, and the general solution of (19) is

$$
f(x)=\alpha x^{2(1-a)}+\beta x^{2}+\gamma x^{2(1+a)} .
$$

From (16) and (20),

$$
F=C_{1}\left(\alpha x^{1-a} y^{1-a}-\gamma x^{1+a} y^{1+a}\right) .
$$

Having satisfied the conditions expressed by (13) and (14), the last member of (12) vanishes and the first member becomes an exact differential, whence integration and substitution of $f$ and $F$ from (20) and (21) give

$$
\frac{x_{\bigvee}\left(\alpha y^{2(1-a)}+\beta y^{2}+\gamma y^{2(1+a)}\right)+y \sqrt{ }\left(\alpha x^{2(1-a)}+\beta x^{2}+\gamma x^{2(1+a)}\right)}{\alpha x^{1-a} y^{1-a}-\gamma x^{1+a} y^{1+a}}=C,
$$

which is an integral of

(23) $\frac{d x}{\sqrt{ }\left(\alpha x^{2(1-a)}+\beta x^{2}+\gamma x^{2(1+a)}\right)}+\frac{d y}{\sqrt{ }\left(\alpha y^{2(1-a)}+\beta y^{2}+\gamma y^{2(1+a)}\right)}=0$.

Making $a=1$, or replacing $x^{a}$ by $x$, and $y^{a}$ by $y$, which may be shown to have the same effect, gives what may be considered the standard forms

$$
\begin{gathered}
\frac{x \sqrt{ }\left(\alpha+\beta y^{2}+\gamma y^{4}\right)+y \sqrt{ }\left(\alpha+\beta x^{2}+\gamma x^{4}\right)}{\alpha-\gamma x^{2} y^{2}}=C, \\
\frac{d x}{\sqrt{ }\left(\alpha+\beta x^{2}+\gamma x^{4}\right)}+\frac{d y}{\sqrt{ }\left(\alpha+\beta y^{2}+\gamma y^{4}\right)}=0 .
\end{gathered}
$$

The denominator in (24) is the integrating factor sought, and is a simple function of $\alpha$ and $\gamma$ in (25), being independent of $\beta$. By giving proper values to $\alpha, \beta$, and $\gamma$ in (24) and (25) addition formulas may be obtained for the logarithm, inverse sine and tangent (both circular and hyperbolic), and elliptic integrals of the first kind. An illustration of the use of the apparently more general forms (22) and (23) is obtained by taking $a=\frac{1}{2}$, then $f(x)$ is $\alpha x+\beta x^{2}+\gamma x^{3}$, which includes $x(1-x)$ $(1-\kappa x)$, favored by Klein as a canonical form in elliptic integrals.

In Schröter's article (loc. cit.) the starting point is

$$
d \phi / \Delta \phi+d \psi / \Delta \psi=0 \quad\left[\Delta \phi=V\left(1-k^{2} \sin ^{2} \phi\right)\right] .
$$

Thus the form of $f(\phi)$ is assigned in advance, and (26) is inte- 
grated by Sturm's method as three distinct problems, each being obtained by a change of variables. Although the Legendrian notation is practically adhered to, the respective substitutions are equivalent to

$$
1-k^{2} \sin ^{2} \phi=x^{2}, \quad \cos ^{2} \phi=x^{2}, \quad \sin ^{2} \phi=x^{2} .
$$

The respective values of $f(x)$ are equivalent to

$$
\left(1-x^{2}\right)\left(x^{2}-k^{\prime 2}\right),\left(1-x^{2}\right)\left(k^{\prime 2}+k^{2} x^{2}\right),\left(1-x^{2}\right)\left(1-k^{2} x^{2}\right)
$$

Schröter's values of $F$ are deduced for each of the three cases independently, by expressing the sufficient conditions for the vanishing of coefficients in his equation corresponding to (12) above. His results are obtainable at once by using his respective values of $f(x)$ from (28) in (25) and (24), and this affords a concrete illustration of the theorem which these two equations express.

University of Pennsylvania, April, 1910.

\section{A PROPERTY OF A SPECIAL LINEAR SUBSTITUTION.}

BY PROFESSOR F. R. SHARPE.

LET $\xi_{i}$ denote homogeneous line coordinates * which satisfy the quadratic identity

$$
\Sigma \xi_{i}^{2}=0 \text {. }
$$

The condition that two lines $\xi$, $\xi^{\prime}$ intersect is

$$
\Sigma \xi_{i} \xi_{i}^{\prime}=0 \text {. }
$$

The equation of a linear complex is of the form

$$
\Sigma a_{i} \xi_{i}=0 \text {. }
$$

It is clear from the above equations that, when

$$
\Sigma a_{i}^{2}=0,
$$

the lines of (3) all intersect the line $a$; (3) is then called a special complex.

* Jessop: Treatise on the line complex, Arts 9, 15-20. 